\title{
E1 Papel de la Mentalización en Psicoterapia para Adolescentes
}

\section{Rubén Antonio Díaz Hernández ${ }^{1 *}$}

${ }^{1}$ Universidad de Chile y Pontificia Universidad Católica de Chile, Profesor, Escuela de Psicología, Universidad Católica Santa María La Antigua (USMA), Panamá, República de Panamá.

*Autor para correspondencia. Email: rubendiazh@gmail.com

Recibido: 15 de agosto de 2018 Aceptado: 30 de octubre de 2018

\section{Resumen}

La mentalización ha sido propuesta como un elemento importante para los procesos psicoterapéuticos. En algunos grupos de pacientes, como los pacientes adolescentes, podría suponerse que el impacto de la mentalización en psicoterapia puede ser más importante por su relación con el logro de tareas evolutivas. La literatura revela evidencias que apoyan parcialmente el rol de la mentalización como moderador, mediador y contenido del cambio en psicoterapia con adultos. Este artículo explorará la evidencia con respecto al papel de la mentalización como mecanismo de cambio en psicoterapia para adolescentes con problemas relacionados a la difusión de identidad, con la premisa de que una mayor comprensión de cómo ocurren los cambios terapéuticos puede optimizar la forma en que los tratamientos se llevan a cabo.

Palabras clave: Función Reflexiva, Mecanismo de Cambio, Investigación de Procesos Psicoterapéuticos, Psicoterapia, Adolescente.

\begin{abstract}
Mentalization has been proposed as an important element for psychotherapeutic processes. In some patient groups, such as adolescent patients, it could be assumed that the impact of mentalization in psychotherapy may be more important because of its relation to the achievement of evolutionary tasks. The literature reveals evidence that partially supports the role of mentalization as moderator, mediator and content of change in psychotherapy with adults. This article will explore the evidence regarding the role of mentalization as a mechanism of change in psychotherapy for adolescents with problems related to the diffusion of identity, with the premise that a greater understanding of how therapeutic changes occur can optimize the way in which treatments are carried out.

Keywords: Reflective Function, Mechanism of Change, Psychotherapeutic Process Research, Psychotherapy, Adolescent.
\end{abstract}


Invest. pens. crit. (ISSN 1812-3864)

Vol. 6, No. 3, septiembre - diciembre 2018

pp. $47-57$

\section{Introducción}

En la tradición de investigación del proceso psicoterapéutico, el interés se ha centrado en estudiar cuál es el cambio psicoterapéutico—contenido—y cómo se produce-factores y mecanismos (Krause \& Altimir, 2016). Para abordar estas preguntas, ha sido muy utilizado el modelo genérico de psicoterapia (Orlinsky \& Howard, 1986), que permite identificar el papel y la interrelación de distintos elementos de la psicoterapia, sus participantes y el contexto.

Desde su introducción el concepto de mentalización ha generado nuevas perspectivas para la comprensión tanto de la psicoterapia como del desarrollo psicológico, y se han desarrollado múltiples tratamientos con la mentalización como componente central (Bateman \& Fonagy, 2012). Un número plural de estudios han arrojado evidencia sobre las características de la mentalización en personas con patologías mentales, así como del impacto de la mentalización en psicoterapia con pacientes adultos (Katznelson, 2014). El presente artículo tiene como objetivo explorar qué dice la evidencia sobre el papel de la mentalización como mecanismo de cambio en psicoterapia. Se focaliza particularmente en el caso de adolescentes con problemas relacionados a la difusión de identidad, porque la adolescencia es un período en el que, por la importancia de la formación de identidad, el sistema de apego-y por consiguiente la mentalización-cobra mayor relevancia (Steele, Bate, Nikitiades, \& Buhl-Nielsen, 2015).

\section{Antecedentes}

\section{Identidad y mentalización en la adolescencia}

Desde la tradición psicodinámica, la identidad ha sido un elemento central para la comprensión del funcionamiento psíquico. La identidad puede definirse como un principio organizador, que se desarrolla a lo largo de la vida, que provee un sentido de continuidad del sí mismo y que sirve como marco para diferenciarse de los otros y funcionar autónomamente (Erikson, 1968/1994). Según Erikson, la tarea principal del desarrollo en la adolescencia es la consolidación de la identidad. Además, en la adolescencia se pone en juego el sistema de apego como base para la formación de identidad, relaciones con los pares, representaciones corporales y el desarrollo de autonomía (Steele et al., 2015). En el caso más extremo, cuando se falla en la tarea de la consolidación de la identidad, los adolescentes pueden perder la capacidad para auto-definirse, experimentan una sensación dolorosa de incoherencia y vacío crónico, presentan comportamientos contradictorios, tienen baja tolerancia a la ansiedad y bajo control de impulsos, así como una falta de compromiso con metas, valores y relaciones (Foelsch et al., 2014). Estas son las características de la difusión de identidad, en cuya base está la falta de una representación integrada de sí mismo, diferenciada de los otros.

Si la difusión de identidad permanece sin diagnóstico y tratamiento, puede permanecer en la adultez, comprometiendo el ajuste psicosocial de los individuos a un funcionamiento bajo (Fonagy et al., 2015). Por ejemplo, la difusión de identidad es uno de los elementos centrales de la organización límite de la personalidad (Kernberg, 1967), siendo esta última un fenómeno que es posible observar de manera temprana en la adolescencia (Winsper et al., 2016). Por otra parte, la mentalización-definida, desde la teoría de apego, como la capacidad para entender el comportamiento propio y de otros en función 
Invest. pens. crit. (ISSN 1812-3864)

Vol. 6, No. 3, septiembre - diciembre 2018

pp. $47-57$

de estados mentales e intenciones subyacentes (Fonagy, Target, \& Gergely, 2000)—juega un papel importante en la difusión de identidad y en el desarrollo de desórdenes de la personalidad (Fonagy, Gergely, Jurist, \& Target, 2004; Fonagy et al., 2000). Sharp y cols. (2011) utilizaron el instrumento Movie for the Assessment of Social Cognition (MASC) para evaluar a 111 pacientes adolescentes y encontraron una relación entre los rasgos límites de personalidad y la hipermentalización (mentalización excesiva e incorrecta).

La capacidad de mentalizar puede desarrollarse en el contexto de un vínculo seguro durante los primeros años de vida, a través de interacciones recurrentes en las que los padres responden de forma adecuada a las emociones del niño. A su vez, esta capacidad se convierte en un factor protector en nuevas situaciones interpersonales, ya que permite considerar múltiples posibilidades sobre las causas del comportamiento del otro, en lugar de adoptar como cierta sólo una conclusión sobre las intenciones del otro (Rothschild-Yakar, Waniel, \& Stein, 2013; Steele et al., 2015; Taubner \& Curth, 2013; Taubner, White, Zimmermann, Fonagy, \& Nolte, 2013).

Considerando que la mentalización puede ser un factor de protección, es comprensible su importancia, no solo en la evolución de la difusión de identidad y los trastornos de personalidad, sino para su tratamiento (Bleiberg, 2013; Forster, Berthollier, \& Rawlinson, 2014; Steele et al., 2015).

\section{Impacto de la mentalización en psicoterapia}

Para la investigación del proceso psicoterapéutico, los diferentes aspectos del paciente, el terapeuta, la interacción y el contexto pueden desempeñar distintas funciones para el cambio. Por ejemplo, Doss (2004) distingue entre procesos de cambio (en psicoterapia y en cliente) y mecanismos de cambio. Menciona que los procesos de cambio son aspectos de la terapia que ocurren durante las sesiones o como resultado directo de asignaciones terapéuticas, que producen mejoras en los mecanismos de cambio; mientras que los mecanismos de cambio hacen referencia a cambios intermedios en las características o habilidades del paciente, que resultan de la terapia y han sido generalizadas a la vida diaria del paciente, y que contribuyen al resultado final de la terapia. A su vez, Kazdin (2007) hace énfasis en que los mecanismos de cambio van más allá de la noción estadística de mediación. Además, identifica el papel de los moderadores del cambio, definiéndolos como características que incluyen en la dirección o magnitud de los efectos de la intervención en el resultado. Finalmente, el contenido del cambio hace referencia a aquello que finalmente cambia en psicoterapia (Krause \& Altimir, 2016). Katznelson (2014) hizo una revisión de la literatura sobre la función reflexiva-como se suele operacionalizar la mentalización para ser estudiada-que incluyó un apartado sobre estudios que evalúan el impacto de la mentalización en psicoterapia. En su revisión, incluyó seis estudios que investigaron procesos psicoterapéuticos de pacientes adultos. Encontró que la evidencia apoya, parcialmente, el papel de la mentalización como moderador, mediador y contenido del cambio psicoterapéutico. En resumen, los estudios revisados muestran, de manera parcial, que:

1. La función reflexiva modera el logro de resultados en terapia, siendo que los pacientes con mayor nivel de función reflexiva pueden beneficiarse de la terapia más rápidamente.

2. Existe una correlación positiva entre la función reflexiva y la alianza terapéutica.

3. Dependiendo del enfoque terapéutico, al final de la terapia se observa un incremento de la función reflexiva, mientras que en otros se mantiene igual o empeora. 
Invest. pens. crit. (ISSN 1812-3864)

Vol. 6, No. 3, septiembre - diciembre 2018

pp. 47-57

4. Los cambios sintomáticos no necesariamente dependen de un incremento en la función reflexiva.

Estos resultados_que a veces contradicen las hipótesis formuladas para el papel de la mentalización en psicoterapia-deben ser interpretados a la luz de las diferencias en las metodologías y fuentes de información para evaluar la mentalización como resultado y como proceso de la psicoterapia.

\section{Formulación del problema}

Si bien la mentalización ha sido considerada un elemento importante para la psicoterapia (Bleiberg, 2013; Shirk \& Burwell, 2010; Steele et al., 2015), la evidencia encontrada hasta el momento no respalda de forma concluyente el impacto que ella ni el papel que juega en el logro del cambio terapéutico. Las revisiones sobre el particular se han realizado con estudios de psicoterapias con pacientes adultos. Es importante rescatar lo que la evidencia dice al respecto del papel de la mentalización en psicoterapia con adolescentes.

En primer lugar, la psicoterapia con adolescentes tiene características que la hacen un fenómeno distinto de su contraparte con adultos: en general, los adolescentes presentan dificultades en el establecimiento de la relación de ayuda; asisten motivados por otros y con baja conciencia de la disfuncionalidad de sus conductas; y algunas características propias de la edad (necesidad de autonomía, autodeterminación y autoconfirmación, desconfianza de la autoridad adulta y desarrollo cognitivo a nivel de operaciones formales) pueden convertirse en un desafío para el trabajo clínico (Fernández-González, Herrera-Salinas, \& Escobar-Martínez, 2016).

Por otra parte, la mayoría de los trastornos mentales de la adultez tienen su inicio en la infancia (Russell, 2008). Además, la adolescencia puede considerarse como una oportunidad evolutiva para reconstruir y reparar modos de funcionamiento psicológico (Erikson, 1968/1994). En el caso particular de los rasgos límites de la personalidad, el tratamiento durante la adolescencia puede ser más efectivo que durante la adultez (Hawes, 2014; Lenzenweger \& Castro, 2005).

Por tanto, en el presente artículo se revisan algunas investigaciones que han incorporado la mentalización como variable de proceso o resultado en el estudio de intervenciones psicoterapéuticas con adolescentes, enfatizando aquellas enfocadas al trabajo con adolescentes problemas relacionados a la difusión de identidad.

\section{Discusión}

\section{Mentalización como contenido del cambio}

Sharp y cols. (2013) se propusieron evaluar si, luego de una intervención terapéutica con adolescentes, se observaría una reducción en la hipermentalización. Para ello, reclutaron 217 adolescentes que participaban en un programa intrahospitalario. De la muestra, $64.5 \%$ presentaba criterios de trastornos internalizantes; $56.5 \%$ de trastornos externalizantes; y $41 \%$ de trastorno límite de la personalidad. Evaluaron la mentalización al momento de ingreso al tratamiento y al momento del alta, empleando 
Invest. pens. crit. (ISSN 1812-3864)

Vol. 6, No. 3, septiembre - diciembre 2018

pp. $47-57$

el instrumento Movie for the Assessment of Social Cognition (MASC). Los resultados que encontraron aportaron evidencia para la hipótesis planteada: hubo una reducción importante y estadísticamente significativa de la hipermentalización al finalizar el tratamiento para la muestra total. Sin embargo, encontraron una interacción de los síntomas de trastorno límite de la personalidad con la hipermentalización: ante la presencia de trastorno límite de la personalidad, la reducción de la hipermentalización fue mayor. Además, encontraron una reducción en la pseudomentalización.

Cryan y Quiroga (2016) evaluaron la evolución de la mentalización en adolescentes violentos que participaron en un Grupo de Terapia Focalizada, una intervención de corto plazo y orientación psicodinámica. Esperaban encontrar diferencias en la función reflexiva de los adolescentes al inicio y al final del tratamiento, evaluada aplicando la Escala de Función Reflexiva a las transcripciones de la primera y última sesión. Los resultados no aportan evidencia para la hipótesis: no hubo cambios en la función reflexiva de los adolescentes cuando se compararon las sesiones. Además, las puntuaciones obtenidas oscilaban entre -1 y 1 , que corresponden a rechazo y ausencia de mentalización. Cabe destacar que el procedimiento para aplicar la escala de Función Reflexiva no es del todo claro en este estudio y esto podría ser una limitante importante, ya que pareciera que se aplicó a nivel de grupo y no a nivel individual. Al respecto, es importante notar que los primeros intentos de estudiar la mentalización aplicando la Escala de Función Reflexiva en sesiones de psicoterapia con adultos (i.e. Karlsson \& Kermott, 2006) tampoco lograron encontrar cambios de la mentalización a lo largo del proceso de psicoterapia.

D'Onofrio, Pace y Cavanna (2015) reportan un estudio de caso de una joven diagnosticada con anorexia nerviosa, paciente en una psicoterapia psicodinámica con frecuencia de dos veces por semana durante dos años. Utilizan la Entrevista de Apego para Niñez y Adolescencia (AICA, por sus siglas en inglés) para evaluar apego y función reflexiva antes y después de iniciar el tratamiento. En su estudio, proponen que el aumento en la función reflexiva es un resultado en sí mismo de la terapia. Aplicando la Escala de Función Reflexiva (Fonagy y cols., 1998) a las entrevistas AICA, encuentran un aumento en el nivel de función reflexiva: desde una puntuación de 1 (RF ausente) en la entrevista previa al tratamiento, hacia una puntuación de 5 (ordinaria) en la entrevista después del tratamiento.

Bo y cols. (2017) estudiaron el efecto de un tratamiento psicoterapéutico grupal basado en mentalización en una muestra de 34 adolescentes en Dinamarca. De los participantes, 25 pacientes completaron el tratamiento-psicoterapia de grupo basada en mentalización para adolescentes-y 9 se retiraron. El tratamiento se llevó a cabo ambulatoriamente en tres clínicas psiquiátricas. La medida primaria fue el Borderline Personality Features Scale for Children, mediante el cual pretendían evaluar si el tratamiento reducía dimensiones del trastorno límite de la personalidad. Entre las medidas secundarias, incluyeron el Reflective Function Questionnaire for Youth (RFQ-Y), un cuestionario de autorreporte dirigido a medir la capacidad general para mentalizar. Se encontró que el tratamiento logró reducir las dimensiones del trastorno límite y aumentar la capacidad general de mentalización en los pacientes.

Puede observarse que los cuatro estudios se han centrado en evaluar intervenciones orientadas desde teorías psicodinámicas. En psicoterapia con adultos, solamente en un tratamiento (Levy et al., 2006) orientado psicodinámicamente se observó un aumento de la función reflexiva como resultado del tratamiento. Faltarían más estudios con adolescentes para evaluar la replicabilidad de los resultados encontrados, así como estudios que incluyan otro tipo de tratamientos. 
Invest. pens. crit. (ISSN 1812-3864)

Vol. 6, No. 3, septiembre - diciembre 2018

pp. 47-57

\section{Mentalización como mecanismo de cambio}

A través de un estudio controlado aleatorizado Rossouw y Fonagy (2012), compararon la efectividad de un tratamiento para adolescentes basado en mentalización (MBT-A) y el tratamiento usual, con un grupo de adolescentes con conductas autolíticas. Además de la medida primaria de resultado, que fue la conducta autolítica, consideraron la mentalización y el apego como posibles mecanismos de cambio. La mentalización la evaluaron con el cuestionario How I Feel (HIF); mientras que el apego, con el Experience of Close Relationships Inventory (ECR). Trabajaron con una muestra de 80 pacientes, 40 asignados a cada rama del estudio. Encontraron que el tratamiento basado en mentalización (MBT-A) logró mayores reducciones en conducta autolítica y riesgo a los 12 meses. Por otra parte, la evidencia favoreció la hipótesis de la mentalización como mecanismo de cambio específico en el tratamiento MBT-A: hubo un aumento en la mentalización en el grupo de MBT-A, no así en el grupo de tratamiento usual. Además, el apego también pareciera ser un mecanismo de cambio específico, según se refleja en los resultados; se logró una reducción en la evitación del apego en el grupo MBT-A y no sigue el mismo patrón en el grupo de tratamiento usual. Además, se verificó el criterio de mediación de Baron y Kenny, tanto para la mentalización como para el apego.

\section{Mentalización como moderador de cambio}

El estudio de Braehler y Schwannauer (2012) no se enmarca dentro de la línea de investigación de procesos de psicoterapia, pero puede arrojar luces sobre el papel de la mentalización como moderador de cambio. Ellos realizaron un estudio cualitativo en el que exploraron la adaptación al primer episodio psicótico. Realizaron entrevistas (una entrevista abierta y la Entrevista de Apego Adulto) a ocho jóvenes y las analizaron que habían sido atendidos por servicio de salud mental adolescente para primer episodio psicótico. Al explorar la relación entre los temas emergentes del análisis de contenido de las entrevistas abiertas con la función reflexiva_calculada a partir de la Entrevista de Apego Adultoencontraron que la función reflexiva moderada aparecía en los casos que presentaron una individuación exitosa y una adaptación adecuada posterior al episodio psicótico. Por otra parte, una baja función reflexiva fue asociada con una adaptación no resuelta y un bloqueo en la individuación después del episodio psicótico. Este resultado está en la misma dirección que lo hallado por Gullestad y cols. $(2013 ; 2011)$ en psicoterapia con adultos.

\section{Consideraciones sobre la investigación de mentalización en psicoterapia con adolescentes}

Los estudios que abordan el impacto de la mentalización en psicoterapia para adolescentes son escasos. Esto impide establecer respuestas concluyentes respecto a la pregunta central de investigación.

Por otra parte, en la mayoría de los artículos revisados se encuentran algunas limitaciones, principalmente metodológicas, principalmente, muestras pequeñas y diseños de investigación débiles (i.e. sin grupo control). Además, si bien varios utilizan la escala de Función Reflexiva-considerada como el gold standard — otros optan por utilizar cuestionarios de autorreporte o tests situacionales.

Aun así, los resultados son promisorios: la evidencia apunta en la dirección de la mentalización como un mecanismo del cambio psicoterapéutico y como un cambio en sí mismo. Se plantea la necesidad de continuar explorando estas diferencias con programas de investigación adaptados a los tratamientos particulares, de manera tal que los diseños sean más sensibles para detectar mecanismos de cambio específicos (Fonagy, 2010; Sharp et al., 2009; Shirk \& Burwell, 2010; Silberschatz, 2017).

Una línea de trabajo que está comenzando a tomar fuerza en la investigación de mentalización como un proceso de cambio en psicoterapia con adultos es la del estudio de la mentalización a partir de las 
Invest. pens. crit. (ISSN 1812-3864)

Vol. 6, No. 3, septiembre - diciembre 2018

pp. $47-57$

sesiones de terapia. Actualmente, existen grupos que investigan las fluctuaciones en la mentalización durante la sesión de psicoterapia (Martínez et al., 2017; Talia et al., 2015; Talia, Miller-Bottome, \& Daniel, 2017; Tomicic, de la Cerda, Martínez, \& Pérez, 2017). Esta línea, inspirada en el trabajo iniciado por Karlsson y Kermott (2006) busca medir la función reflexiva a partir de lo que los pacientes dicen en el marco de una sesión terapéutica, en contraposición con la evaluación de la función reflexiva a partir de narrativas en entrevistas o de cuestionarios de autorreporte. Esta nueva forma de medir puede arrojar luces sobre la forma como se va construyendo el cambio en la mentalización durante las sesiones (por ejemplo, qué intervenciones del terapeuta favorecen una mayor función reflectiva en el paciente y en qué momentos de la relación terapéutica y del tratamiento).

Además, es interesante considerar la mentalización en el período evolutivo de estudio. La mentalización — como capacidad fluctuante y dependiente del contexto relacional—podría encontrar también bloqueos o facilitadores en otros contextos adicionales a la psicoterapia. Entonces, valdría la pena incorporar, por ejemplo, una mirada a las formas en que los padres de los adolescentes favorecen u obstaculizan los cambios en la capacidad de mentalización del adolescente y, por ende, su posibilidad de impactar en el resultado terapéutico.

\section{Conclusiones}

Aún existen pocos estudios para brindar respuestas concluyentes sobre el papel de la mentalización en psicoterapia con adolescentes. Sin embargo, hasta el momento los resultados arrojan resultados en cuanto a que la mentalización puede considerarse tanto un cambio en sí mismo, como un mecanismo de cambio en las muestras estudiadas.

\section{Referencias}

Bateman, A., \& Fonagy, P. (2012). Handbook of mentalizing in mental health practice. Washington, DC: American Psychiatric Pub.

Bleiberg, E. (2013). Mentalizing-based treatment with adolescents and families. Child and Adolescent Psychiatric Clinics of North America, 22(2), 295-330. https://doi.org/10.1016/j.chc.2013.01.001

Bo, S., Sharp, C., Beck, E., Pedersen, J., Gondan, M., \& Simonsen, E. (2017). First empirical evaluation of outcomes for mentalization-based group therapy for adolescents with BPD. Personality Disorders: Theory, Research, and Treatment, 8(4), 396-401. https://doi.org/10.1037/per0000210

Braehler, C., \& Schwannauer, M. (2012). Recovering an emerging self: Exploring reflective function in recovery from adolescent-onset psychosis. Psychology and Psychotherapy: Theory, Research and Practice, 85(1), 48-67. https://doi.org/10.1111/j.20448341.2011.02018.x 
Invest. pens. crit. (ISSN 1812-3864)

Vol. 6, No. 3, septiembre - diciembre 2018

pp. $47-57$

Cryan, G., \& Quiroga, S. (2016). Analisis de la función reflexiva-RF en sesiones de grupo de terapia focalizada para adolescentes violentos. Interamerican Journal of Psychology, 50(2). Recuperado a partir de http://www.redalyc.org/html/284/28447010010/

D’Onofrio, E., Pace, C. S., \& Cavanna, D. (2015). Qualitative research in adolescent psychotherapy: attachment and reflective functioning as psychotherapy's outcomes of an adolescent with anorexia nervosa. Research in Psychotherapy: Psychopathology, Process and Outcome, 18(2). https://doi.org/10.4081/ripppo.2015.181

Doss, B. D. (2004). Changing the way we study change in psychotherapy. Clinical Psychology: Science and Practice, 11(4), 368-386. https://doi.org/10.1093/clipsy/bph094

Erikson, E. H. (1994). Identity, youth and crisis. New York: Norton. Publicado originalmente en 1968.

Fernández-González, O. M., Herrera-Salinas, P., \& Escobar-Martínez, M. J. (2016). Adolescentes en psicoterapia: Su representación de la relación terapéutica. Revista Latinoamericana de Ciencias Sociales, Niñezy Juventud, 14(1). Recuperado a partir de http:// revistaumanizales.cinde.org.co/index.php/RevistaLatinoamericana/article/view/238 6

Foelsch, P. A., Schlüter-Müller, S., Odom, A. E., Arena, H. T., Borzutzky H, A., \& Schmeck, K. (2014). Adolescent Identity Treatment: An integrative approach for personality pathology. Cham, Suiza: Springer International Publishing.

Fonagy, P. (2010). The changing shape of clinical practice: ¿Driven by science or by pragmatics? Psychoanalytic Psychotherapy, 24(1), 22-43. https://doi.org/10.1080/02668731003590139

Fonagy, P., Gergely, G., Jurist, E., \& Target, M. (2004). Affect regulation, mentalization, and the development of the self. Londres, Inglaterra: Karnac Books.

Fonagy, P., Speranza, M., Luyten, P., Kaess, M., Hessels, C., \& Bohus, M. (2015). ESCAP Expert Article: Borderline personality disorder in adolescence: An expert research review with implications for clinical practice. European Child \& Adolescent Psychiatry, 24(11), 1307-1320. https://doi.org/10.1007/s00787-015-0751-z

Fonagy, P., Target, M., \& Gergely, G. (2000). Attachment and borderline personality disorder: A theory and some evidence. Psychiatric Clinics of North America, 23(1), 103-122. https://doi.org/10.1016/S0193-953X(05)70146-5

Forster, C., Berthollier, N., \& Rawlinson, D. (2014). A systematic review of potential mechanisms of change in psychotherapeutic interventions for personality disorder. Journal of Psychology \& Psychotherapy, 04(01). https://doi.org/10.4172/21610487.1000133

Gullestad, F. S., Johansen, M. S., Høglend, P., Karterud, S., \& Wilberg, T. (2013). Mentalization as a moderator of treatment effects: Findings from a randomized clinical trial for personality disorders. Psychotherapy Research, 23(6), 674-689. 
Invest. pens. crit. (ISSN 1812-3864)

Vol. 6, No. 3, septiembre - diciembre 2018

pp. $47-57$

https://doi.org/10.1080/10503307.2012.684103

Gullestad, F. S., \& Wilberg, T. (2011). Change in reflective functioning during psychotherapy- A single-case study. Psychotherapy Research, 21(1), 97-111. https://doi.org/10.1080/10503307.2010.525759

Hawes, D. J. (2014). Does the concept of borderline personality features have clinical utility in childhood? Current Opinion in Psychiatry, 27(1), 87-93. https://doi.org/10.1097/YCO.0000000000000018

Karlsson, R., \& Kermott, A. (2006). Reflective-functioning during the process in brief psychotherapies. Psychotherapy: Theory, Research, Practice, Training, 43(1), 65-84. https://doi.org/10.1037/0033-3204.43.1.65

Katznelson, H. (2014). Reflective functioning: A review. Clinical Psychology Review, 34(2), 107-117. https:/ / doi.org/10.1016/j.cpr.2013.12.003

Kazdin, A. E. (2007). Mediators and mechanisms of change in psychotherapy research. Annual Review of Clinical Psychology, 3(1), 1-27. https://doi.org/10.1146/annurev.clinpsy.3.022806.091432

Kernberg, O. (1967). Borderline personality organization. Journal of the American Psychoanalytic Association, 15(3), 641-685.

Krause, M., \& Altimir, C. (2016). Introduction: current developments in psychotherapy process research / Introducción: desarrollos actuales en la investigación del proceso psicoterapéutico. Estudios de Psicologia, 37(2-3), 201-225. https://doi.org/10.1080/02109395.2016.1227574

Lenzenweger, M. F., \& Castro, D. D. (2005). Predicting change in borderline personality: Using neurobehavioral systems indicators within an individual growth curve framework. Development and Psychopathology, 17(4), 1207-1237. https://doi.org/10.1017/S0954579405050571

Levy, K. N., Clarkin, J. F., Yeomans, F. E., Scott, L. N., Wasserman, R. H., \& Kernberg, O. F. (2006). The mechanisms of change in the treatment of borderline personality disorder with transference focused psychotherapy. Journal of Clinical Psychology, 62(4), 481- 501. https://doi.org/10.1002/jclp.20239

Martínez, C., Tomicic, A., de la Cerda, C., Rivera, M. J., Salas, C., Pontificia Universidad Católica de Chile, ... Hospital Sótero del Río, Santiago de Chile. (2017). Función reflexiva en primeras entrevistas de psicoterapia: Un estudio exploratorio sobre el papel de la mentalización en la interacción terapéutica. Revista Argentina de Clinica Psicologica, 26(2). https://doi.org/10.24205/03276716.2017.1026 
Invest. pens. crit. (ISSN 1812-3864)

Vol. 6, No. 3, septiembre - diciembre 2018

pp. $47-57$

Orlinsky, D. E., \& Howard, K. I. (1986). Process and outcome in psychotherapy. En S. Garfield \& A. Bergin (Eds.), Handbook of psychotherapy and behavior change ( $3^{a}$ ed., pp. 311- 381). New York: Wiley.

Rossouw, T. I., \& Fonagy, P. (2012). Mentalization-based treatment for self-harm in adolescents: A randomized controlled trial. Journal of the American Academy of Child \& Adolescent Psychiatry, 51(12), 1304-1313.e3. https://doi.org/10.1016/j.jaac.2012.09.018

Rothschild-Yakar, L., Waniel, A., \& Stein, D. (2013). Mentalizing in self vs. parent representations and working models of parents as risk and protective factors from distress and eating disorders. The Journal of Nervous and Mental Disease, 201(6), 510-518.

https://doi.org/10.1097/NMD.0b013e3182948316

Russell, R. L. (2008). Child and adolescent psychotherapy research: Introduction to the special section. Psychotherapy Research, 18(1), 1-4. https://doi.org/10.1080/10503300701725090

Sharp, C., Ha, C., Carbone, C., Kim, S., Perry, K., Williams, L., \& Fonagy, P. (2013).

Hypermentalizing in adolescent inpatients: Treatment effects and association with borderline traits. Journal of Personality Disorders, 27(1), 3-18. https://doi.org/10.1521/pedi.2013.27.1.3

Sharp, C., Pane, H., Ha, C., Venta, A., Patel, A. B., Sturek, J., \& Fonagy, P. (2011). Theory of mind and emotion regulation difficulties in adolescents with borderline traits. Journal of the American Academy of Child and Adolescent Psychiatry, 50(6), 563-573.e1. https://doi.org/10.1016/j.jaac.2011.01.017

Sharp, C., Williams, L. L., Ha, C., Baumgardner, J., Michonski, J., Seals, R., ... Fonagy, P. (2009). The development of a mentalization-based outcomes and research protocol for an adolescent inpatient unit. Bulletin of the Menninger Clinic, 73(4), 311-338. https://doi.org/10.1521/bumc.2009.73.4.311

Shirk, S., \& Burwell, A. (2010). Research on therapeutic processes: in psychodynamic psychotherapy with children and adolescents. Assessing Change in Psychoanalytic Psychotherapy of Children and Adolescents: Today's Challenge, 177-205.

Silberschatz, G. (2017). Improving the yield of psychotherapy research. Psychotherapy Research, 27(1), 1-13. https://doi.org/10.1080/10503307.2015.1076202

Steele, M., Bate, J., Nikitiades, A., \& Buhl-Nielsen, B. (2015). Attachment in adolescence and borderline personality disorder. Journal of Infant, Child, and Adolescent Psychotherapy, 14(1), 16-32. https://doi.org/10.1080/15289168.2015.1004882

Talia, A., Miller-Bottome, M., \& Daniel, S. I. F. (2017). Assessing attachment in psychotherapy: Validation of the Patient Attachment Coding System (PACS). Clinical Psychology \& Psychotherapy, 24(1), 149-161. https://doi.org/10.1002/cpp.1990 
Invest. pens. crit. (ISSN 1812-3864)

Vol. 6, No. 3, septiembre - diciembre 2018

pp. $47-57$

Talia, A., Miller-Bottome, M., Svenja, T., Katznelson, H., Lunn, S., Pedersen, S. H., ... Daniel, S. I. F. (2015). Mentalizing in the presence of another: Validating the patient attachment coding system as a measure of reflective functioning in psychotherapy. En Society for Psychotherapy Research, 46th Annual International Meeting.

Taubner, S., \& Curth, C. (2013). Mentalization mediates the relation between early traumatic experiences and aggressive behavior in adolescence. Psibologija, 46(2), 177-192. https://doi.org/10.2298/PSI1302177T

Taubner, S., White, L. O., Zimmermann, J., Fonagy, P., \& Nolte, T. (2013). Attachment-related mentalization moderates the relationship between psychopathic traits and proactive aggression in adolescence. Journal of Abnormal Child Psychology, 41(6), 929-938. https://doi.org/10.1007/s10802-013-9736-x

Tomicic, A., de la Cerda, C., Martínez, C., \& Pérez, C. (2017). Reflective functioning scale in relevant episodes: Moving forward to an interactional comprehension of mentalizing in the psychotherapy process. Presentado en 48th International Annual Meeting SPR, Toronto, Canada.

Winsper, C., Lereya, S. T., Marwaha, S., Thompson, A., Eyden, J., \& Singh, S. P. (2016). The aetiological and psychopathological validity of borderline personality disorder in youth: A systematic review and meta-analysis. Clinical Psychology Review, 44(Supplement C), 13-24. 